\title{
anu \\ Cyclic Tension Induced Pattern Formation on [001] Single-Crystal Aluminum Foil
}

\author{
Kuznetsov Pavel *(D) and Khon Yury $(D)$ \\ Institute of Strength Physics and Materials Science SB RAS, 634055 Tomsk, Russia; khon@ispms.tsc.ru \\ * Correspondence: kpv@ispms.tsc.ru
}

\begin{abstract}
Cyclic tension of (100)[001]-oriented single-crystal aluminum foils with the frequency $5 \mathrm{~Hz}$ forms a tweed pattern. Its period is several microns and increases by a factor of 1.5 in the temperature range $233-363 \mathrm{~K}$. A model is proposed for structural relaxation of the medium on spatial and time meso- and macroscales under cyclic loading. Conditions under which a steady pattern forms are found based on the analysis of kinetic equations. The number of bands in the steady pattern is found to be related to the strain rate. The process activation energy is determined.
\end{abstract}

Keywords: cyclic deformation; aluminum foil; tweed structure; structural relaxation; activation energy

\section{Introduction}

Investigations into cyclic deformation of pure fcc metals play an important role in elucidating the fundamental laws of fatigue fracture initiation and propagation in metals [1-3]. These investigations proceed in the three interrelated directions: the cyclic stress-strain (CSS) curves, evolution of dislocation structures, and surface slip morphology [1-3]. The best-studied crystals are copper, nickel, and silver [1]. It can be noted, that in most cases, cyclic deformation is subjected to a qualitative study because the quantitative characterization poses methodological difficulties.

At present, the cyclic deformations of single crystals of silver, copper, and nickel with low, average, and high stacking fault energy are best understood. The CSS curves for the single slip-oriented crystals at the controlled plastic strain amplitude in the range $\Delta \varepsilon \sim\left(10^{-4}-10^{-2}\right)$ have a plateau after the stage of primary cyclic hardening. This plateau corresponds to the formation of ladder-like Persistent Slip Bands (PSBs), whose fractions grow due to a decrease in the fraction of the surrounding matrix [1-3]. The observed regularities can be described quantitatively within the two-phase model [4]. PSBs on the crystal surface are the sites of extrusions and intrusions as well as fatigue crack initiation [2].

Cyclic deformation of $\mathrm{Cu}, \mathrm{Ni}$, and $\mathrm{Ag}$ crystals oriented for double and multiple slip at the controlled plastic strain amplitude is characterized by a higher primary hardening rate than that of the single slip-oriented crystals. As the plastic strain amplitude grows, the CSS curve can have either an extended plateau, a short plateau, or a continuous increase without a plateau and saturation depending on the single crystal orientation [5-8]. PSB ladder-like structures are usually parallel to active slip systems, but PSB ladders can correspond to secondary slip systems at higher plastic strain amplitude [9]. At $\gamma_{\mathrm{pl}}>2.5 \cdot 10^{-3}$, type-I and type-II deformation bands appear on the specimen due to accommodation of the rotational deformation mode [8]. Therefore, crystals with double and multiple slip orientation have more diverse cyclic response curves, dislocation structures, and surface slip morphologies as compared to single slip-oriented crystals.

According to the study of the temperature effect on the cyclic deformation of differently oriented copper single crystals, a lower test temperature leads to an increase in saturation stress and a systematic decrease in the PSB wall period [10,11]. It was shown [2] that the saturation stress under cyclic deformation corresponds to the flow stress at the stage of parabolic hardening for copper single crystals. The investigation of the PSB cross sections 
and shapes of the corresponding extrusions and intrusions on the specimen surface reveals their dependence on the temperature [11,12].

The behavior of aluminum single crystals under cyclic deformation, whose stacking fault energy is the highest of fcc crystals, differs strongly from that of copper, nickel, and silver crystals [13-16]. Differences appear in CSS curves, dislocation ordering, and surface morphology. CSS curves for aluminum single crystals under room temperature cyclic deformation have no plateau at any controlled plastic strain amplitude [14]. It is replaced by the "primary cyclic hardening-softening-secondary cyclic hardening" sequence [14]. PSB ladders typical of copper, nickel, and silver are not observed on cyclically-deformed aluminum single crystals at room temperature, and dislocation ordering has mainly a cellular structure regardless of orientation $[13,16]$.

The slip band pattern in aluminum has important differences from that in copper, nickel, and silver: PSBs do not propagate through the crystal cross section, but small PSB segments are clustered on the active slip plane [13].

Under cyclic deformation of aluminum single crystals with the [001] orientation, a specific structure is formed on the crystal surface, which appears as a regular network of fine lines in the optical microscope and is referred to as a tweed structure [14]. According to the detailed study [15], this structure presents spherical protrusions on the aluminum surface, which are formed by diagonal lines at an angle of $45^{\circ}$ to the loading axis and whose characteristics are independent of the controlled plastic strain amplitude and the number of cycles. The authors of [15] pointed to an important feature of the fracture of specimens: "Most of the runs had to be terminated due to a collapse of the crystals and corresponding break-down of the control over the plastic strain amplitude. Fatigue failure in the traditional meaning in which cracks are formed and one of these grows to a size that lead to the ultimate failure was not observed".

A similar structure was also observed on polycrystalline pure aluminum foils glued to flat specimens of high-strength aluminum alloy under cyclical deformation at room temperature [17] and $77 \mathrm{~K} \mathrm{[18].} \mathrm{The} \mathrm{tweed} \mathrm{structure} \mathrm{can} \mathrm{be} \mathrm{observed} \mathrm{in} \mathrm{some} \mathrm{bulk} \mathrm{polycrystalline}$ aluminum grains [19].

The dislocation structure of aluminum single crystals and polycrystals after cyclic deformation was studied in a number of works to establish a direct correlation between the tweed structure on the surface and the underlying dislocation structure [15,18]. According to [15], at the stage of cyclic softening and secondary cyclic hardening, when the cord and tweed structures form on foils cut parallel to the surface, there appear dislocation walls (001). In some areas they form simultaneously, resulting in a labyrinth-like structure. Similar wall structures, predominantly with the single set of $\{100\}$ walls, were observed in polycrystalline aluminum foils after cyclic deformation at the temperature $77 \mathrm{~K} \mathrm{[18].}$

The formation of wall structures in fatigued FCC metals is fully explained by the (double pseudo-polygonization) model proposed in [20]. The model predicts the highest probability of (001) walls for [001] single crystals and the equal probabilities of (100) and (010) walls. In accordance with the model, the (001) walls can accommodate all eight slip systems activated under high-symmetry loading of a single crystal. The remaining $\{100\}$ walls can accommodate only dipole loops in the four expected slip systems.

The study of foils cut from the inner regions of crystals [15] showed that, at the stage of secondary cyclic hardening, an ordered system of spherical dislocation subcells with a low density of dislocations in them is formed on the (001) dislocation walls. This indicates an increase in the dislocation density in the walls at the stage of secondary cyclic hardening and the formation of subcells at a critical level [15].

From the analysis it was found [16] that $\{100\}$ dislocation wall structures observed on foils cut from different crystallographic sections correspond to a series of weak lines on the specimen surface, parallel and perpendicular to the tension axis. However, the correspondence between the dislocation structure of the walls and the tweed structure on the surface remains unclear [15]. 
According to [18], the tweed structure formation depends on the presence of a significant number of dislocations with four distinct Burgers vectors, which requires a tension axis close to [001]. To investigate the dislocation structure in relation to surface deformation features, one side of the foils was electropolished [18]. Based on the analysis of the investigation results, the tweed structure is suggested to form as a result of the extrusion process [18], in which the material surrounding the tweed structure protrusions is relatively soft. Thus, the analysis of the literature data on the relationship between the tweed structure on the aluminum crystal surface and the underlying dislocation structure shows that this issue remains open and requires further investigation.

The study of the temperature effect on the cyclic deformation of aluminum single crystals showed that a decrease in the test temperature to $77 \mathrm{~K}$ increases the saturation stress and results in a plateau in the CSS curves [14]. The dislocation structure formed at $77 \mathrm{~K}$ was similar to the two-phase structure formed in single slip-oriented copper under cyclic deformation, though no ladder-like structure was observed in this case [14].

The independence of the tweed structure period on the controlled plastic strain and the number of cycles [15] allows for a quantitative study of the temperature effect on the cyclic deformation of aluminum single crystals.

The aim of this work is to study the effect of temperature on the tweed structure formation during constrained cyclic tension of [001]-oriented single-crystal $\mathrm{Al}$ foils and to develop a theoretical model of macroscopic deformation and pattern formation.

\section{Materials and Methods}

As a substrate, we used specimens of grade D1 duralumin in the form of dumbbells with the gauge section $60 \mathrm{~mm} \times 10 \mathrm{~mm} \times 2 \mathrm{~mm}$. Duralumin specimens were mechanically polished using pastes of different dispersion. Foils were prepared from plates measuring $16 \mathrm{~mm} \times 20 \mathrm{~mm} \times 0.5 \mathrm{~mm}$, which were cut from an Al single crystal with orientation (100)[001] by the electroerosive method. Both sides of the plates were mechanically polished to a thickness of about 250-270 microns on polishing paper with a gradual decrease in the grain size of the abrasive to $3-5$ microns, and then electrochemically polished in $74 \mathrm{~mL}$ $\mathrm{N}_{2} \mathrm{SO}_{4}, 74 \mathrm{~mL} \mathrm{H}_{3} \mathrm{PO}_{4}, 16 \mathrm{~g} \mathrm{CrO}_{3}, 56 \mathrm{~mL} \mathrm{H}_{2} \mathrm{O}$ electrolyte up to the thickness $200 \mu \mathrm{m}$. The foils were glued to the central part of the duralumin specimen surface using Loctite 480 glue.

Duralumin specimens were tested for low-cycle fatigue at the temperatures 233, 263, $296,313,333$, and $363 \mathrm{~K}$ with the following parameters: frequency $f=5 \mathrm{~Hz}, \sigma_{\max }=165 \mathrm{MPa}$, $\sigma_{\min }=0.1 \sigma_{\max }$, and $\sigma_{\text {mean }}=\left(\sigma_{\max }-\sigma_{\min }\right) / 2$. Loading was performed using a UTM150 servohydraulic testing machine (BISS (P) Ltd.) with a PAC-70-B-EUR-RRU-INT climatic chamber (CM Envirosystems (P) Ltd.). In the first 10 cycles at $1 \mathrm{~Hz}$, the stress amplitude was elevated gradually from 0 to $165 \mathrm{MPa}$. Then, the frequency was brought to $5 \mathrm{~Hz}$. The number of test cycles $N=5000$ was the same for all specimens. After testing, the specimens were removed from the testing machine and the surface of single-crystal Al foils was examined through an Axiovert 25CA optical microscope.

\section{Results}

The surface of single-crystalline aluminum foils after cyclic tension demonstrates a tweed structure, which was earlier observed on foils [17] and bulk specimens [15] of (100)[001]-oriented aluminum single crystals at room temperature. This pattern appears on single-crystalline aluminum foils soon after the onset of cyclic deformation without visible slip lines, which conforms to the previous studies of cyclic deformation of bulk specimens and foils of aluminum single crystals $[15,17]$. The formation of a pattern on the foils is accompanied by the appearance of an interference shade, visible through the window of the climatic chamber [17]. The visible interference shade is used to estimate the formation time of the tweed structure $t$. The time varies from $t_{1}=(600 \pm 60) \mathrm{s}$ in cyclic tests at $T_{1}=233 \mathrm{~K}$ to $t_{2}=(200 \pm 30) \mathrm{s}$ at the temperature $T_{2}=363 \mathrm{~K}$. 
Figure 1 exemplifies optical images of the tweed structure formed at the temperatures $T=233,296$, and $363 \mathrm{~K}$. It can be seen that an increase in the test temperature leads to a noticeable increase in the tweed structure period.

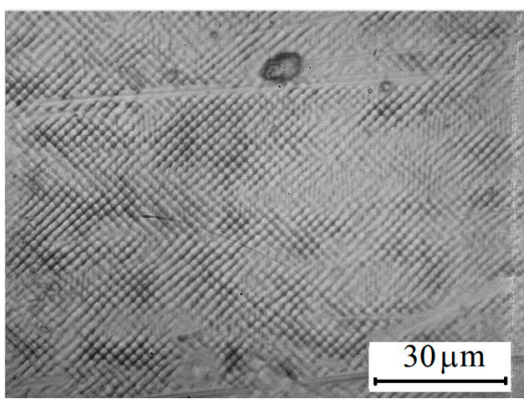

(a)

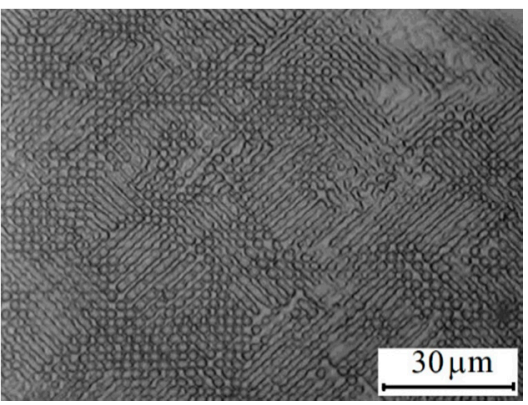

(b)

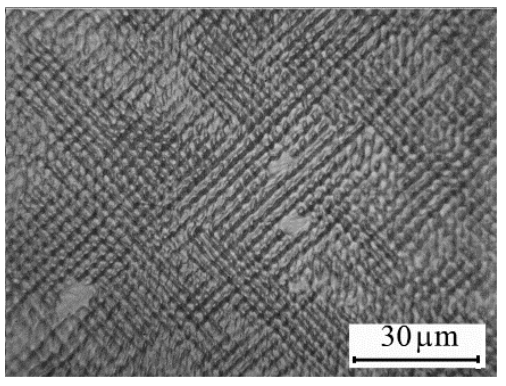

(c)

Figure 1. Tweed structure on (100)[001] Al single crystal foils after cyclic deformation at temperatures of $233 \mathrm{~K}(\mathbf{a}), 296 \mathrm{~K}(\mathbf{b})$, and $363 \mathrm{~K}(\mathbf{c})$.

To obtain quantitative information, optical images of the tweed structure are statistically processed and histograms are constructed for each test temperature.

Figure 2 shows histograms for the temperatures 233, 296, and 363K. From the histograms, it can be seen that the average period of the tweed structure increases with increasing temperature. This indicates that the tweed structure formation during cyclic tension is a thermally-activated process. From the tweed structure period histograms, follow the average period $(R)$ and the standard deviation $\Delta R$ for each test temperature. The average period of the tweed structure on the interval $\Delta \mathrm{T}=(233 \div 363) \mathrm{K}$ increases from $R_{1}=(1.92 \pm 0.12) \mu \mathrm{m}$ to $R_{2}=(3.03 \pm 0.15) \mu \mathrm{m}$, i.e., by almost $50 \%$. Figure 3 plots the dependence of $\ln \left(\frac{R}{R_{1}}\right)$ on $1 / \mathrm{T}$, which approximates well to a straight line.

This indicates that thermally-activated processes of structural relaxation determine the tweed structure formation on single-crystalline aluminum foils under cyclic tension.

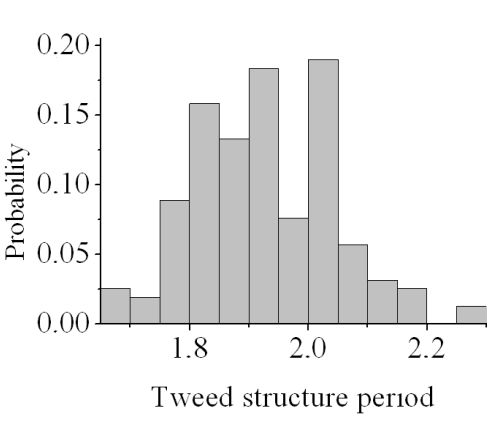

(a)

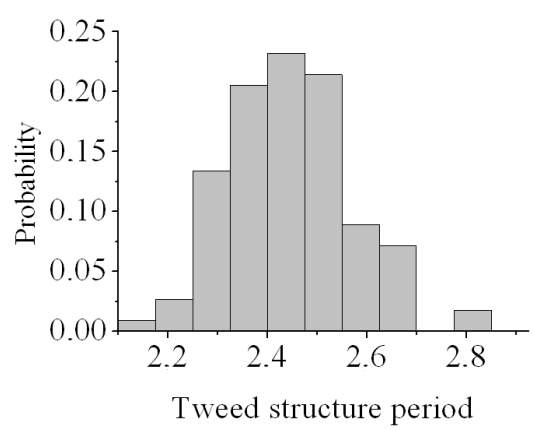

(b)

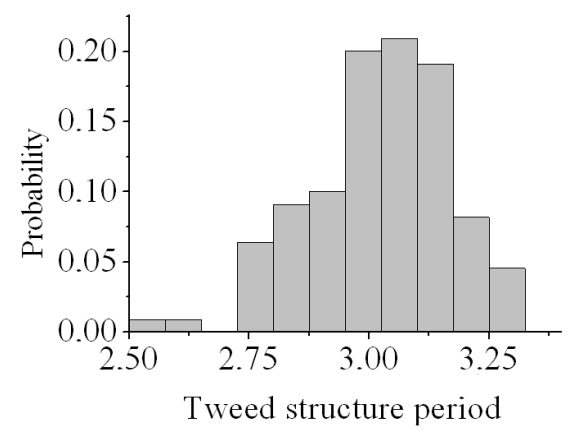

(c)

Figure 2. Histograms of the tweed structure period formed at temperatures of 233 (a), 296 (b) and 363 (c) K. 


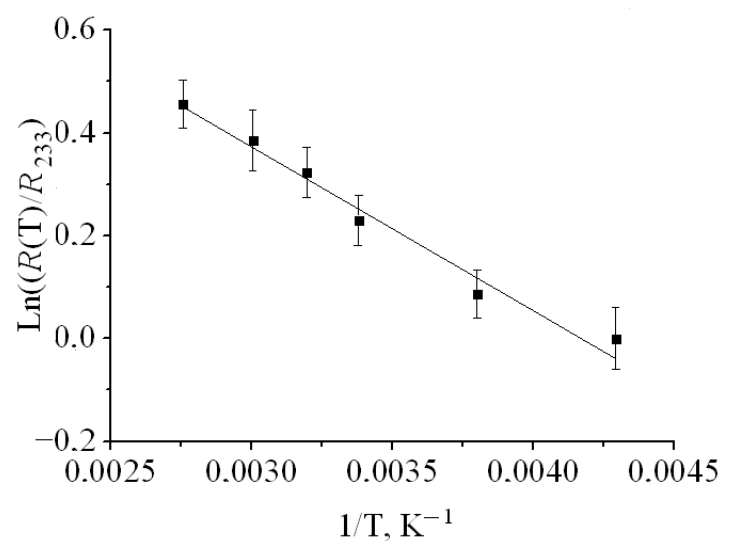

Figure 3. Dependence of the logarithm of the reduced tweed structure period on the reciprocal temperature.

\section{Cyclic-Induced Pattern Formation Model}

Let us now dwell on factors determining plastic deformation localization and conditions under which the periodic pattern forms. Dislocation mechanisms of plastic deformation of aluminum at the stage of parabolic hardening due to double cross slip of dislocations are known. However, dislocation mechanisms alone are inadequate to describe macroscopic deformation. There are two reasons for this. First, consideration should be given not only to dislocations, as stated above, but also to point defects. Second, structural relaxation of the system under plastic deformation is determined by the nucleation and interaction of deformation carriers on all spatial and time scales. However, not only direct solutions but also the writing of dynamic equations is hardly possible for deformation carriers. Other approaches are required to take into account the relaxation processes on large spatial and time scales. A phenomenological approach was proposed to solve the problem [21]. This approach can be used to study the regularities of nucleation and propagation of localized deformation bands in the form of traveling fronts at the stage of easy slip and linear hardening. In this work, the developed approach and the macroscopic deformation model are applied towards the solution of the pattern formation problem during the cyclic tension of foils of aluminum single crystals with cubic orientation.

We study the deformation of a flat specimen, which is cyclically extended to $\varepsilon(t)$ along axis $x$ with frequency $f$ under applied stress $\sigma$. The specimen plane lies in the plane $z=0$ of the Cartesian coordinate system $x, y, z$. A medium under deformation is assumed to be homogeneous and isotropic. The one-dimensional case is considered.

Plastic deformation is a process of structural relaxation determined by the nucleation and motion of deformation carriers under an external force on all spatial $l_{i}$ and time $t$ scales ( $i$ is the scale number). By the scale, we mean the unstable-mode wavelength $\lambda_{i} \sim l_{i}$ with the frequency $\omega_{i} \sim \frac{1}{t_{i}}$ of correlated displacements of inelastic deformation carriers. Structural relaxation of a deformed medium on larger scales is determined by processes on smaller scales. Consideration is given to two spatial and time scales: $l_{1}<l_{2}, t_{1}<t_{2}$. In the experiments, we measure the displacement distribution related to the long-wave mode on the scale $l_{2} \sim 1 \mu \mathrm{m}$. The short-wave mode is determined by displacements on scales less than $l_{1}$.

According to [21], structural changes in a deformed medium can be described by dynamic order parameters $\varphi(x, t)$ and $\eta(x, t)$. These functions have the meaning of a volume fraction with structural changes leading to the excitation and development of two deformation modes on scales $l_{1}$ and $l_{2}$, respectively. Local plastic strain in the linear approximation is written in the form

$$
\varepsilon^{p}(x, t)=\bar{\varepsilon}_{S} \varphi(x, t)+\bar{\varepsilon}_{L} \eta(x, t)
$$


Here, $\bar{\varepsilon}_{S}, \bar{\varepsilon}_{L}$ are the parameters determined by the mechanisms and conditions of the medium deformation on the scales $l_{1}, l_{2}$, respectively. Deformation modes at which

$$
\left\langle\dot{\varepsilon}_{S}(x, t)+\dot{\varepsilon}_{L}(x, t)\right\rangle \dot{\varepsilon}(t)
$$

appear in experiments. Here, angular brackets stand for averaging over the specimen length. At each time instant, deformation modes that decrease the elastic energy of the system are excited and develop.

Kinetic equations for $\varphi$ and $\eta$ have the form [22]

$$
\begin{gathered}
t_{2} \partial_{t} \eta=(-r+p \varphi) \eta-b \eta^{3}+l_{2}^{2} \partial_{x}^{2} \eta \\
t_{1} \partial_{t} \varphi=(\alpha-g \eta) \varphi+q_{2} \varphi^{2}-q_{3} \varphi^{3}+l_{1}^{2} \partial_{x^{2}}^{2} \varphi
\end{gathered}
$$

Here, $\alpha, g, q_{2}, q_{3}, b, p$ are the parameters determined by plastic deformation carriers. The parameter $\alpha$ depends on the applied load (elastic strain $\varepsilon_{e l}$ ) and may alternate in signs, the rest of the parameters are positive. The quantity $r=\frac{\varepsilon_{\mathcal{C}}-\varepsilon_{e l}}{\varepsilon_{c}}>0$ is the dimensionless threshold of stability of the medium at $\varphi=0$. The deformation carrier nucleation $(\varphi>0)$ lowers the stability threshold of the system and initiates structural relaxation of the medium on larger scales. When the temperature rises, $r$ for aluminum decreases.

By introducing the variables

$$
\widetilde{t}_{2}=t_{2} / r, \widetilde{l}_{2}=l_{2} / r^{1 / 2}, \widetilde{\eta}=\eta b^{\frac{1}{2}} r^{-1 / 2}, \widetilde{\varphi}=\varphi q_{3}{ }^{1 / 2}
$$

Equations (3) and (4) can be reduced to (the sign " $\sim$ " is further omitted)

$$
\begin{gathered}
t_{2} \partial_{t} \eta=(-1+d \varphi) \eta-\eta^{3}+l_{2}^{2} \partial_{x}^{2} \eta \\
t_{1} \partial_{t} \varphi=\alpha \varphi-c \eta \varphi+\beta \varphi^{2}-\varphi^{3}+l_{1}^{2} \partial_{x}^{2} \varphi
\end{gathered}
$$

Here

$$
\beta=q_{2} q_{3}^{-\frac{1}{2}}, d=p q_{3}^{-\frac{1}{2}} / r, c=g b^{-\frac{1}{2}} r^{1 / 2}
$$

The system of two coupled nonlinear parabolic Equations (6) and (7) describes structural relaxation of a deformed medium on two spatial and time scales. The governing parameter in these equations is elastic strain. Let us explain the physical meaning of Equations (6) and (7). At $\varphi=0$, Equation (6) has a unique stable homogeneous solution $\eta_{0}=\eta=0$, which describes elastic deformation of the medium. Plastic strain is determined by a change in the internal structure on a smaller scale, which occurs at $\varphi>0$. The parameter $d$ in (6) depends on the strain rate $\dot{\varepsilon}$ of the specimen: $d$ increases with an increase in $\dot{\varepsilon}$. Equation (7) describes the structural changes on smaller scales, which are determined by the nucleation and development of ensembles of interacting carriers of irreversible deformation. At $\eta=0, E(7)$ has two homogeneous stationary solutions $\varphi_{0}=\varphi=0$ and $\varphi_{h}=\frac{\beta}{2}+\left(\frac{\beta^{2}}{4}+\alpha\right)^{1 / 2}$. The solution $\varphi_{0}$ describes the elastically deformed state of the medium. The solution $\varphi_{\mathrm{h}}$ describes the state of the medium with structural changes. It is stable at $\alpha>-2 \beta^{2} / 9$. With consideration for the stress dependence of the parameters $\alpha$ and $\beta$, the ratio $-2 \frac{\beta^{2}}{9}=\alpha$ gives the threshold stress above which irreversible displacements are excited in the medium. At $\alpha>0$, the solution $\varphi_{0}$ is unstable to small heterogeneous perturbations. The pattern formation is preceded by a uniform deformation. Therefore, the parameter $\alpha>0$. The presence of the term $-c \eta \varphi$ on the right side of (7) means that mesoscopic plastic deformation is accompanied by an increase in the elastic energy of the system.

Equations (6) and (7) always have a homogeneous stationary solution $\eta_{0}=\eta=0$, $\varphi_{0}=\varphi=0$. Stationary homogeneous solutions $\varphi_{h}>0, \eta_{h}>0$ present the intersection points of the curves

$$
\eta=(-1+d \varphi)^{1 / 2}
$$




$$
\eta=\left(\alpha+\beta \varphi-\varphi^{2}\right) / c
$$

The analysis of the solutions of these equations and their stability to small perturbations shows the following. Stationary solutions $\varphi_{h}>0, \eta_{h}>0$ can be unstable to small heterogeneous perturbations at

$$
d>d_{c}=2\left[c^{2}+\left(\alpha+\frac{\beta^{2}}{4}\right)^{2}\right] / \beta c^{2}
$$

At $d=d_{c}$, curve (9) intersects curve (10) at its maximum point $\varphi=\varphi_{m}=\beta / 2$. From (11), it can be seen that increasing $c$ reduces $d_{c}$ and contributes to deformation localization. Instability to small heterogeneous perturbations develops when the inequalities

$$
\tau<1, l \ll 1, \tau<l
$$

are valid. Here

$$
\tau=\frac{t_{1}}{t_{2}}, l=\frac{l_{1}}{l_{2}}
$$

With Equation (13), the third inequality in (12) reduces to the condition $v_{\eta}>v_{\varphi}$ The second inequality in (12) means that deformation carriers should be in short-range interaction with each other and have a shorter characteristic time of excitation. This explains the fact that structural relaxation at the stage of parabolic hardening is determined by double cross slip of dislocations and the generation of point defects. However, the physical aspect of its influence on macrodeformation localization is poorly understood within the theory of defects.

\section{Discussion}

Solutions to Equations (6) and (7) describing the periodic pattern formation are analyzed by numerical methods for the one-dimensional case. The initial and boundary conditions are given in Appendix A. The analysis of the numerical solutions shows that the pattern of spatial structures is almost completely determined by $l_{2}$ in (6) and $l$ in (13). On higher $l_{2}$ at a constant value $l$, the spacing between localized deformation bands increases. As an example, Figures 4 and 5 show the spatial distributions of the dynamic order parameters at

$$
\alpha=0.03, \beta=0.8, d=10, l_{1} / l_{2}=0.05, \tau=0.8 c=0.4
$$

and on different $1_{2}$. At the parameters used in $(14), \eta_{h} \approx 0.26, \varphi_{h} \approx 0.11, d_{c} \approx 3.1$. The distribution of the dynamic order parameters calculated for $l_{2}=1.4$ is shown in Figure 4 .

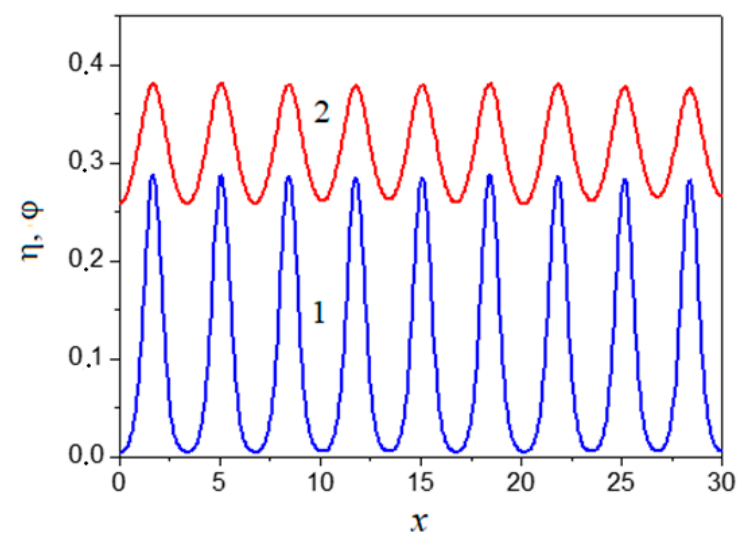

Figure 4. Spatial distribution of the variables $\varphi$ (curve 1) and $\eta$ (curve 2) on $l_{2}=1.4$. 


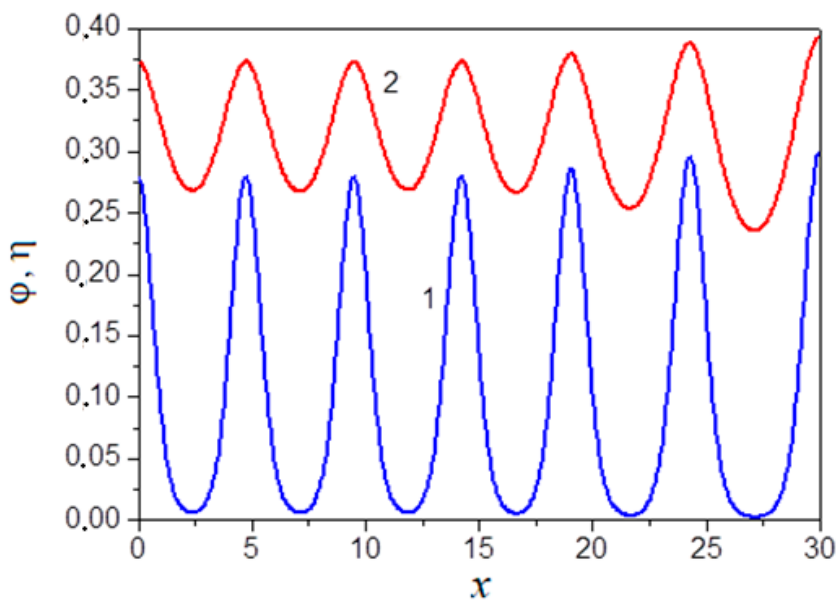

Figure 5. Spatial distribution of the variables $\varphi$ (curve 1) and $\eta$ (curve 2) on $l_{2}=2.1$.

It can be seen that plastic deformation is nonuniformly distributed along the specimen length. Nine equally spaced striations are formed. In so doing, $\left.\langle\varphi\rangle \approx \varphi_{h},\langle\eta\rangle \approx 0.31\right\rangle$ $\eta_{h}$. On $l_{2}=2.1$, six localized deformation bands are formed (Figure 5), as in Figure 4, $\langle\varphi\rangle \approx \varphi_{h},\langle\eta\rangle \approx 0.31>\eta_{h}$. With increasing temperature, the parameter $r$ decreases, and $l_{2}$, according to (5), increases. Therefore, the band spacing increases with temperature, which agrees with the experiment.

As indicated above, the spacing between localized deformation bands is almost completely determined by the value $l_{2} \sim 1 / r^{1 / 2}$ in (6). The higher $l_{2}$ is, the greater $R$ is. This means that the $r$ value should decrease as the temperature rises. The linear dependence $\ln \left(\frac{\mathrm{R}}{\mathrm{R}_{1}}\right) \sim-1 / \mathrm{T}$ (Figure 3) means that $n\left(r_{1} / r_{2}\right) \sim-1 /$ T, i.e., the dimensionless threshold of stability decreases with increasing temperature, which is in qualitative agreement with the experimental data.

Plastic deformation is a thermally-activated process. Let us express strain rates at the temperatures $\mathrm{T}_{1}, \mathrm{~T}_{2}$ as $\dot{\varepsilon}_{1}\left(\mathrm{~T}_{1}\right) \sim \exp \left(-\frac{E}{k \mathrm{~T}_{1}}\right), \dot{\varepsilon}_{2}\left(\mathrm{~T}_{2}\right) \sim \exp \left(-\frac{E}{k \mathrm{~T}_{2}}\right)$, respectively. Here, $E$ is the activation energy at the stage of uniform deformation (preceding the instability), and $k$ is the Boltzmann constant. Under cyclic tension, the strain of the specimen remains constant. Then the equality

$$
t_{1} \dot{\varepsilon}_{1}\left(\mathrm{~T}_{1}\right)=t_{2} \dot{\varepsilon}_{2}\left(\mathrm{~T}_{2}\right)
$$

holds. The activation energy found from (15) equals $E \approx 0.07 \mathrm{eV}$. This value is lower than the migration activation enthalpy of interstitial atoms in aluminum $E_{m}^{i} \approx 0.12 \mathrm{eV}$ [22]. At the same time, $E$ is higher than the activation energy of dislocation slip. It should be noted that no model underlies the estimate of the activation energy, but it stems from the analysis of kinetic equations of the formation of a stationary pattern and the relationship between the strain rate and the number of bands. The analysis of results based on the Einstein diffusion model [23] gave the migration activation energy of interstitial aluminum atoms $E_{m}^{i} \approx 0.12 \mathrm{eV}$.

The results obtained show that the tweed structure formation on single-crystalline $\mathrm{Al}$ foils under cyclic tension in the temperature range $\Delta \mathrm{T}=(233 \div 363) \mathrm{K}$ is controlled by the slowest process, i.e., migration of interstitial atoms. This is an unexpected result since a small number of interstitial atoms (as compared to vacancies) form in fcc metals due to the much higher energy of formation, and the probability of their annihilation is high. In addition, interstitial atoms have low migration activation energy and high mobility. Therefore, they are thought to be completely annealed at temperatures of several tens of Kelvin.

The above arguments are very weighty, and an increase in the role of interstitial atoms within point defect kinetics remains currently unexplained. The role of point defects in mass redistribution and surface relief formation during cyclic deformation and solidification of 
crystals has been intensively investigated [2,12,24-26]. The model of extrusion development due to a high density of vacancy defects was proposed in [24] based on the knowledge of dislocation ordering in PSBs and the experimental study of the rate of point defect generation in cyclically-deformed crystals. A model taking into account the generation of vacancies and interstitial atoms, both in PSBs and in the matrix, was developed in [25]. Consideration for the generation of interstitial atoms during cyclic hardening made it possible to explain the formation and shape of extrusions and intrusions at very low temperatures when vacancy defects are inactive. The role of point defects in the surface relief formation was studied mainly for copper and stainless steel crystals oriented for easy slip, in which deformation localization can occur in PSBs.

\section{Conclusions}

1. The experimental and theoretical results show that the periodic pattern formation on [001]-oriented single-crystalline aluminum foils under cyclic deformation is controlled by the slow, as compared to the dislocation velocity, process of structural relaxation of the medium, which is characterized by a low activation energy comparable to the migration activation energy of interstitial aluminum atoms.

2. In the proposed model of pattern formation, the structural relaxation of a deformed medium is considered on two different spatial and time scales and is described by two small parameters (dynamic order parameters). This description of structural changes is independent of the specific mechanisms of plastic deformation. The kinetics of structural relaxation of a deformed medium is described by a system of two coupled nonlinear parabolic equations with parameters depending on the temperature and strain rate of the specimen.

3. It is shown that a periodic pattern develops under the following conditions. First, the homogeneous state should be unstable to finite-amplitude perturbations of dynamic order parameters. Second, the characteristic relaxation time on smaller spatial scales should be shorter than that on larger scales. Third, structural changes on the mesoscopic scale should be short range.

4. Consideration for the low activation energy of structural relaxation of the medium is likely to explain all differences in the aluminum behavior under cyclic deformation from other pure fcc metals.

Author Contributions: Conceptualization, K.P. and K.Y.; methodology, K.Y.; validation, K.P.; formal analysis, K.Y.; investigation, K.P.; resources, K.P. and K.Y.; data curation, K.P.; writing-original draft preparation, K.Y.; writing-review and editing, K.P. and K.Y.; visualization, K.P. and K.Y.; supervision, K.P.; project administration, K.Y.; funding acquisition, K.P. All authors have read and agreed to the published version of the manuscript.

Funding: The work was performed according to the government research assignment for ISPMS SB RAS, project topics number FWRW-2021-0011 and FWRW-2021-0009.

Data Availability Statement: The data presented in this study are available on request from the corresponding author. The data are not publicly available due to a proper institutional repository policy.

Acknowledgments: The authors would like to express their gratitude to S.V. Panin for providing access to their instrumentation and A. Byakov for his assistance in the implementation of the cyclic test.

Conflicts of Interest: The authors declare no conflict of interest. The funders had no role in the design of the study; in the collection, analyses, or interpretation of data; in the writing of the manuscript, or in the decision to publish the results.

\section{Appendix A}

Numerical solutions of the system of Equations (6) and (7) are found under periodic boundary conditions. The initial conditions are set to $\varphi(x, t=0)=0.11, \eta(x, t=0)=0.26$. 
The initial perturbation of the variables $\varphi$ and $\eta$ is assumed to be stochastic with the amplitude $0 \leq \Delta \eta(x)=\Delta \varphi(x) \leq 10^{-2}$.

\section{References}

1. Li, P.; Li, S.; Wang, Z.; Zhang, Z. Fundamental factors on formation mechanism of dislocation arrangements in cyclically deformed fcc single crystals. Prog. Mater. Sci. 2011, 56, 328-377. [CrossRef]

2. Basinski, Z.; Basinski, S. Fundamental aspects of low amplitude cyclic deformation in face-centred cubic crystals. Prog. Mater. Sci. 1992, 36, 89-148. [CrossRef]

3. Sauzay, M.; Kubin, L. Scaling laws for dislocation microstructures in monotonic and cyclic deformation of fcc metals. Prog. Mater. Sci. 2011, 56, 725-784. [CrossRef]

4. Winter, A.T. A model for the fatigue of copper at low plastic strain amplitudes. Philos. Mag. 1974, 30, 719-738. [CrossRef]

5. Lepistö, T.; Kettunen, P. Comparison of the cyclic stress-strain behaviour of single-and $<111>$ multiple-slip-oriented copper single crystals. Mater. Sci. Eng. 1986, 83, 1-15. [CrossRef]

6. Gong, B.; Wang, Z.; Wang, Z. Cyclic deformation behavior and dislocation structures of [001] copper single crystals-I Cyclic stress-strain response and surface feature. Acta Mater. 1997, 45, 1365-1377. [CrossRef]

7. Wang, Z.; Gong, B.; Wang, Z. Cyclic deformation behavior and dislocation structures of [001] copper single crystals-II. Characteristics of dislocation structures. Acta Mater. 1997, 45, 1379-1391. [CrossRef]

8. Li, X.; Wang, Z.; Li, G.; Wu, S.; Li, S. Cyclic stress-strain response and surface deformation features of [011] multiple-slip-oriented copper single crystals. Acta Mater. 1998, 46, 4497-4505. [CrossRef]

9. Li, P.; Zhang, Z.; Li, X.; Li, S.; Wang, Z. Effect of orientation on the cyclic deformation behavior of silver single crystals: Comparison with the behavior of copper and nickel single crystals. Acta Mater. 2009, 57, 4845-4854. [CrossRef]

10. Holzwarth, U.; Essmann, U. Temperature-induced rearrangement of the dislocation pattern of Persistent Slip Bands in copper single crystals. Appl. Phys. A 1994, 58, 197-210. [CrossRef]

11. Basinski, Z.; Korbel, A.; Basinski, S. The temperature dependence of the saturation stress and dislocation substructure in fatigued copper single crystals. Acta Met. 1980, 28, 191-207. [CrossRef]

12. Polák, J. Production, annihilation and migration of point defects in cyclic straining. Materials 2020, 14, 100938. [CrossRef]

13. Vorren, O.; Ryum, N. Cyclic deformation of al-single crystals at low constant plastic strain amplitudes. Acta Met. 1987, 35, 855-866. [CrossRef]

14. Vorren, O.; Ryum, N. Cyclic deformation of Al single crystals: Effect of the crystallographic orientation. Acta Met. 1988, 36, 1443-1453. [CrossRef]

15. Videm, M.; Ryum, N. Cyclic deformation of [001] aluminium single crystals. Mater. Sci. Eng. A 1996, 219, 1-10. [CrossRef]

16. Li, P.; Li, S.; Wang, Z.; Zhang, Z. Cyclic Deformation Behaviors of \{5\}79] Oriented Al Single Crystals. Met. Mater. Trans. A 2010, 41, 2532-2537. [CrossRef]

17. Kuznetsov, P.V.; Petrakova, I.V.; Gordienko, Y.G.; Zasimchuk, E.E.; Karbovskii, V.L. Formation of self-similar structures on (100)[001] aluminum single-crystal foils under cyclic tension. Phys. Mesomech. 2009, 12, 85-93. [CrossRef]

18. Charsley, P.; Harris, L. Condensed dislocation structures in polycrystalline aluminium fatigued at 77K. Scr. Met. 1987, 21, 341-344. [CrossRef]

19. Videm, M.; Ryum, N. Cyclic deformation and fracture of pure aluminium polycrystals. Mater. Sci. Eng. A 1996, 219, 11-20. [CrossRef]

20. Dickson, J.; Boutin, J.; L’Espérance, G. An explanation of labyrinth walls in fatigued F.C.C. metals. Acta Met. 1986, 34, 1505-1514. [CrossRef]

21. Khon, Y.; Zuev, L. Plastic flow viewed as a process of space-temporal structure formation. Part II. Generation and devel-opment of localized structures: Two level macroscopic description. Phys. Mesomech. 2022, 25, in print.

22. Wollenberger, H.J. Point defects. In Physical Metallurgy, 4th ed.; Cahn, R.W., Haasen, P., Eds.; North-Holland Physics Publishing House: Amsterdam, The Netherlands; Oxford, UK; New York, NY, USA; Tokyo, Japan, 1996; Volume 2, pp. $1622-1721$.

23. Kuznetsov, P.; Panin, S.V.; Byakov, A.V. Temperature impact on tweed structure formation on aluminum single-crystal foil surface under cyclic tension. AIP Conf. Proc. 2018, 2051, 020165. [CrossRef]

24. Essmann, U.; Gösele, U.; Mughrabi, H. A model of extrusions and intrusions in fatigued metals I. Point-defect production and the growth of extrusions. Philos. Mag. A 1981, 44, 405-426. [CrossRef]

25. Polák, J. On the role of point defects in fatigue crack initiation. Mater. Sci. Eng. 1987, 92, 71-80. [CrossRef]

26. Coutinho, J. Theory of the Thermal Stability of Silicon Vacancies and Interstitials in 4H-SiC. Crystals 2021, 11, 167. [CrossRef] 